patients with sickle cell anaemia was shown promptly to decrease the incorporation of radioactive iron into red cells and cause reticulocytopenia and rapid worsening of the anaemia. These events reversed rapidly when administration of oxygen was stopped. ${ }^{2}$ The haematological events in our patient are identical to those observed in that study, which also showed that patients with pernicious anaemia exposed to similar concentrations of oxygen during treatment with vitamin $B_{12}$ had a suboptimal reticulocyte response.

A brief period of red cell hypoplasia is of little consequence to an individual with red cells with a normal life span but will cause a rapid decrease in the packed cell volume in the patient with haemolytic anaemia of any cause. Failure to recognise this iatrogenic cause of aplastic crisis may lead to unwarranted diagnostic procedures and treatment. In the patient with a nutritional anaemia hyperoxia may retard the reticulocyte response to the administration of the deficient nutrient and thereby mislead the physician. Finally, studies of haematological changes during vaso-occlusive crises in patients with sickle cell disease should take account of the potential contribution of hyperoxygenation.

${ }^{1}$ Serjeant GR, Topley JM, Mason K, et al. Outbreak of aplastic crises in sickle cell anemia associated with parvovirus-like agent. Lancet 1981; ii:595-7.

2 Tinsley JC Jr, Moore CV, Dubach R, Minnich V, Grinstein M. The role of oxygen in the regulation of erythropoiesis. Depression of the rate of delivery of new red cells to the blood of high concentrations of inspired oxygen. F Clin Invest 1949;28:1544-64.

(Accepted 24 May 1983)

Haematology Section, Department of Medicine, University of Oklahoma College of Medicine, Oklahoma City, Oklahoma, USA DILIP L SOLANKI, MD, associate professor of medicine

Requests for reprints to: $\mathrm{Dr} D$ L Solanki, Haematology Section (IIIJ), VA Medical Center, $921 \mathrm{NE}$ 13th Street, Oklahoma City, Oklahoma 73104, USA.

\section{Regular reinfusion of ascites during haemodialysis in a patient with amyloidosis}

We describe a patient with primary amyloidosis who had recurrent massive ascites due to hepatic and peritoneal amyloid disease and renal failure requiring haemodialysis. She was treated for 10 months by regular reinfusion of her ascitic fluid into the haemodialysis circuit to relieve massive abdominal distension and prevent hypoproteinaemia and hypotension.

\section{Case report}

A 48 year old woman presented with the nephrotic syndrome and ascites in April 1981. Renal and liver biopsy specimens showed amyloidosis, which on immunofluorescence examination was of the amyloid AL or primary type. There was no evidence of an underlying disease and the plasma creatinine concentration was normal. In August 1981 she developed anuric renal failure; renal vein or inferior vena cava thrombosis was diagnosed clinically, but no radiological confirmation was sought. She started haemodialysis through an arteriovenous shunt and had repeated paracenteses to relieve her painful and disabling abdominal distension. It proved impossible to remove adequate amounts of fluid by ultrafiltration during dialysis, as she was profoundly hypotensive and hypoproteinaemic both during and between dialyses despite frequent infusions of plasma protein fractions. In October 1981 a Tenckhoff permanent peritoneal dialysis catheter was inserted for paracentesis, and a thrice weekly programme of reinfusion of her ascitic fluid during haemodialysis was started. During each dialysis between 2.5 and 4 litres of ascites (protein concentration 20-30 g/l) were drained out and then reinfused through a filter directly into the bubble trap of the dialysis circuit (figure). There were no febrile reactions, no disturbance of blood coagulation, and no episodes of bacteraemia or peritonitis.

Treatment was continued for 10 months, during which time her plasma albumin concentration rose from 22 to $31 \mathrm{~g} / 1$ with only occasional plasma infusions, and her systolic blood pressure rose to $90-110 \mathrm{~mm} \mathrm{Hg}$. Postural and dialysis induced hypotension still occurred from time to time, but with less frequency and severity than at the start of regular haemodialysis, and the patient led a reasonably independent life at home between dialyses. She eventually died from liver failure, having become more jaundiced and encephalopathic over the preceding two months. At necropsy there was severe amyloid infiltration of the liver, kidneys, peritoneum, and heart.

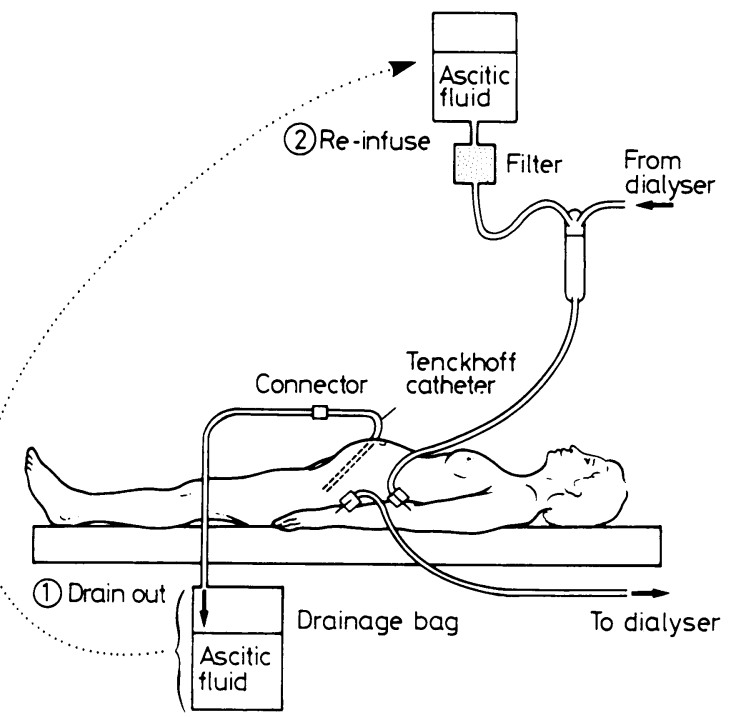

At the start of haemodialysis the Tenckhoff catheter was connected via polyvinylchloride tubing to a standard sterile peritoneal dialysis drainage bag (Travenol 2C4107) (1). The ascites was then reinfused through a blood filter (Viggo Swank transfusion filter 6000-4) into the bubble trap of the dialysis circuit (2). Ultrafiltration during dialysis was adjusted according to the volume of fluid infused and the blood pressure. After dialysis the Tenckhoff catheter was spigoted off and the end bound in povidone-iodine soaked gauze until the next treatment.

\section{Comment}

The management of ascites in the presence of renal failure is a major problem in both amyloidosis and cirrhosis of the liver, and few long term treatments have been described. In the present case ultrafiltration either alone or during haemodialysis was totally ineffective in removing the ascitic fluid, merely resulting in profound hypotension due to hypovolaemia. Insertion of a LeVeen peritoneovenous shunt for recirculation of the ascites has been occasionally reported in patients having haemodialysis, ${ }^{1}$ but the risk of thrombotic or infective complications was felt to be too high in our frail patient.

The method of treatment we have described was chosen as a simple method of returning her ascites to the circulation on a regular basis, and also restored a relatively normal plasma albumin concentration without the excessive use of scarce and costly plasma infusions. Simultaneous dialysis allowed for the correction of any possible hypervolaemia caused by the reinfusion. Our experience over 10 months suggests that the method is an effective way of treating ascites in an anuric patient, with improvement in blood pressure and conservation of plasma proteins.

We thank the nursing staff of the dialysis units of Royal Hallamshire and Lodge Moor Hospitals for their care, cooperation, and technical skill in treating this patient in a novel way.

ADDENDUM-Since we submitted this paper we have treated a further patient with amyloidosis who had identical clinical problems of ascites and end stage renal failure. However, during her first session of reinfusion of ascites during haemodialysis she sustained a fatal cardiac arrest. We have no reason to believe on clinical, laboratory, or necropsy evidence that this patient's death was a complication of the reinfusion procedure.

${ }^{1}$ LeVeen HH, Wapnick S, Grosberg S, Kinney MJ. Further experience with peritoneo-venous shunt for ascites. Ann Surg 1976;184:574-81.

Department of Medicine, Royal Hallamshire Hospital, Sheffield S10 2JF

A J NICHOLLS, MB, MRCP, senior registrar in nephrology

$M$ M PLATTS, MD, FRCP, formerly reader in medicine

D R TRIGER, DPHIL, FRCP, senior lecturer in medicine

Correspondence to: Dr A J Nicholls. 